Exp. Brain Res. 34, 117--132 (1979)

Experimental

\title{
Coding of Small Sinusoidal Frequency and Amplitude Modulations in the Inferior Colliculus of 'CF-FM' Bat, Rhinolophus Ferrumequinum*
}

\author{
G. Schuller
}

Arbeitskreis Neuro- und Rezeptorphysiologie, Fachbereich Biologie, Universität Frankfurt, Siesmayerstr. 70, D - 6000 Frankfurt, Federal Republic of Germany

\begin{abstract}
Summary. Single neurons in the inferior colliculus of the Greater Horseshoe bat, Rhinolophus ferrumequinum, showed two broad categories of response patterns to sinusoidally frequency (SFM) or amplitude (SAM) modulated stimuli. Tonic responding cells (best excitatory frequency (BEF) between 10 and $90 \mathrm{kHz}$ ) showed a rough sinusoidal modulation of the discharge pattern to SFM. Transient responding neurons, generally showing on- or off-responses to pure tones, (BEF between 65 and $88 \mathrm{kHz}$ ), displayed highly synchronized discharge patterns to SFM-cycles (Fig. 1). Modulation rates between 20 and $100 \mathrm{~Hz}$ were most effective and some neurons encoded modulation rates up to $350 \mathrm{~Hz}$ (Figs. 2 and 3). The SFM responses were best synchronized to the modulation envelope for center frequencies in the upper portion of the tuning curve (Figs. 4 and 5). Sharply tuned neurons with BEF around $80 \mathrm{kHz}$ had the lowest threshold for modulation depth $( \pm 10 \mathrm{~Hz}$ or $0.025 \%$ ) (Fig. 6). In general, SAMs evoked the same type of response patterns and were encoded down to modulation index of $3 \%$ (Fig. 7). The fine frequency and amplitude discriminations for periodical modulations by collicular neurons is discussed as related to the detection and discrimination performance of bats, when preying on flying insects in clustered surroundings.
\end{abstract}

Key words: Inferior colliculus - Sinusoidal frequency modulations Sinusoidal amplitude modulations - Bat - Single neurons.

Greater Horseshoe bats, Rhinolophus ferrumequinum, emit orientation cries composed of a long constant frequency (CF) portion (10-60 ms) at frequencies

\footnotetext{
* Supported by Stiftung Volkswagenwerk, AZ. 11858 and Deutsche Forschungsgemeinschaft, SCHU 390/1, 2 and BR 593/2

Offprint requests to: G. Schuller, M.D. (address see above)
} 
between $81-85 \mathrm{kHz}$ and a final frequency modulated portion that sweeps downward by $13-20 \mathrm{kHz}$ in $1-3 \mathrm{~ms}$. The echoes may or may not have the same frequency as the emitted $\mathrm{CF}$ portion; stationary targets reflect an echo at the same frequency as the emitted pulse while moving targets introduce an overall Doppler shift on the CF and FM portions. Of particular interest, the Horseshoe bats manipulate their pulses during echolocation by lowering their emitted frequencies to compensate for Doppler shifts thereby holding the echo $\mathrm{CF}$ component at one particular frequency called the reference frequency (Schnitzler, 1968; Schuller et al., 1974).

Whereas the overall echo frequency is Doppler shifted upward during insect pursuit, the carrier frequency of the echo will also have additional amplitude and frequency modulations produced by the wingbeats of a flying insect. Consequently, the acoustic structure of the echoes received during insect pursuit will be considerably different and more complex than that of the emitted pulses.

It has been suggested that the AM and FM modulations superimposed on the CF portion of the echo are utilized by the bat for the detection and recognition of insects as potential prey (Schuller, 1972, Johnson et al., 1974). Strong support for this hypothesis is provided by Goldman and Henson (1977) who demonstrated that insects are pursued and eaten by Pteronotus parnellii (another long CF-FM bat) only when the insects are flying or are otherwise free to move their wings.

The response features of neurons in Rhinolophus appear to be well suited for processing complex echoes. Neurons with best frequencies in the range of the pulse and echo CF-components are very sharply tuned having $\mathrm{Q}_{10 \mathrm{~dB}}$ values as high as 300 and exhibit dramatic changes in the form of their spike count functions with frequency and amplitude. Sharp tuning coupled with the spike count function behaviour should allow neurons to encode rapid changes in signal intensity and frequency. Preliminary data from Pteronotus reported by Suga and Jen (1977) indicate that sharply tuned auditory cochlear nucleus neurons are sensitive to SFM. Furthermore, evoked potential studies in the inferior colliculus of the Horseshoe bat demonstrate that the evoked potentials clearly follow even small linear frequency modulations, as small as $10 \mathrm{~Hz}(0.012 \%)$ and sweeprates down to $6 \mathrm{~Hz} / \mathrm{ms}$ (Schuller, 1972).

The purpose of the following experiments was to obtain insights into the general response characteristics of single neurons in the inferior colliculus of the Greater Horseshoe bat evoked by sinusoidal frequency and amplitude modulated signals with special interest in the properties of neurons having best frequencies in the range of the constant frequency component of the emitted echolocation sounds.

\section{Methods}

Responses to amplitude and frequency modulated sounds were tested in 8 Greater Horseshoe bats, Rhinolophus ferrumequinum. Under ether anesthesia the skull of the bat was exposed, a screw was mounted with dental cement on its anterior part, small holes were drilled in the skull over the colliculus inferior and the dura was removed for electrode penetrations. After administration of neuroleptic $(4 \mathrm{mg} / 1000 \mathrm{~g}$ droperidol) the animals were put in an animal holder which restricted 
gross body movements and the cemented screw was locked into a rigid metal bar to immobilize the head. During the experiment the wound margins were continuously treated with local anesthetic agents (Novocain).

Acoustic stimuli were presented under open field conditions by a condenser loudspeaker at a distance of $10 \mathrm{~cm}$ to the contralateral ear. The frequency response of the loudspeaker was flat within $1 \mathrm{~dB}$ in the range from $75 \mathrm{kHz}$ to $90 \mathrm{kHz}$ and $3 \mathrm{~dB}$ in the range from $50 \mathrm{kHz}$ to $100 \mathrm{kHz}$. For sound levels at and below $90 \mathrm{~dB}$ SPL the distortions of the loudspeaker output were at least $-40 \mathrm{~dB}$ for frequencies above $30 \mathrm{kHz}$.

Single neuron responses were recorded with glass microelectrodes filled with $3 \mathrm{M}-\mathrm{KCl}$ $(8-20 \mathrm{M} \Omega)$ and alcian blue dye, which allowed electrophoretical marking of two locations in each electrode penetration. After the experiments the animals were sacrificed, the brains were fixed in formalin and histologically processed to identify the electrode path.

The nerve impulses were amplified and processed with conventional methods and fed on-line to a PDP-12 (later a PDP-11) laboratory computer accumulating post-stimulus time histograms. The computer programs for acquisition and processing of the PST-histograms were written by $\mathrm{H}$. Zöller.

The stimuli consisted of ultrasonic pulses of 30-100 ms duration and rise- and fall-times of $2 \mathrm{~ms}$. The sinusoidal modulating waveforms for frequency and amplitude modulations (rectangular and triangular waveforms were occasionally used) were generated by a Wavetek (Model 112), which was phaselocked to the beginning of the stimulus, so that the modulation always started at a precise phase angle. This modulation waveform was applied to the VCG-input of a Wavetek (Model 136) generator for frequency modulations or to one input terminal of the 4-quadrant multiplier built-in to this generator for amplitude modulation. The phases of the modulating waveform and the carrier frequencies had no defined relationship at the beginning of the stimulus. The modulating frequencies for both modulation types were varied between $10 \mathrm{~Hz}$ and $500 \mathrm{~Hz}$. Frequency modulation depth ranged from $\pm 5 \mathrm{kHz}$ to $\pm 10 \mathrm{~Hz}$ and amplitude modulations with modulation indices (defined below) between $100 \%$ to $3 \%$ were tested.

Sinusoidally amplitude modulated signals consist of 3 spectral components: the carrier frequency $f_{0}$ and 2 side frequencies $\left(f_{o} \pm f_{m}\right)$, where $f_{m}$ is the modulating frequency. The modulation index for amplitude modulations is defined as the ratio between the peak amplitude $\left(\mathrm{U}_{\mathrm{m}}\right)$ of the half-wave rectified envelope of the signal and the amplitude of the unmodulated signal $U_{o}\left(m=U_{m} / U_{o}\right)$. The modulation index determines the distribution of energy to the 3 spectral components. The relative amplitude of side frequencies compared to the amplitude of the carrier frequency is $\mathrm{m} / 2$ each. An amplitude modulation of $100 \%(\mathrm{~m}=1)$ means that the energy in each side band is $6 \mathrm{~dB}$ lower than that of the carrier frequency. The spectrum of a frequency modulated signal contains an infinite series of side frequencies $\left(\mathrm{f}_{\mathrm{o}} \pm \mathrm{k} \mathrm{f}_{\mathrm{m}} ; \mathrm{k}=1,2, \ldots\right)$. The distribution of energy to the side frequencies depends on the modulation index, which is defined as the ratio of the maximum instantaneous frequency deviation of of the signal to the modulating frequency $\mathrm{f}_{\mathrm{m}}$. If the frequency modulation depth $\mathrm{df}$ is small compared to the modulating frequency $f_{\mathrm{m}}$ (i.e., the modulation index is much smaller than 1) only the first 2 side frequencies contain considerable energy. Under these conditions the spectrum of the frequency modulated signal resembles that of the amplitude modulated signal, but differs in the relative phase angle of the spectral components. Whenever the modulation index is greater then $1, \mathrm{p}$ spectral components $\left(\mathrm{f}_{\mathrm{o}} \pm \mathrm{k} \mathrm{f}_{\mathrm{m}} ; \mathrm{k}=1,2 \ldots \mathrm{p}\right)$ contribute significantly energy to the frequency modulated signal, where $\mathrm{p}=1+\mathrm{df} / \mathrm{f}_{\mathrm{m}}$. For example, a frequency modulation of $\pm 1200 \mathrm{~Hz}$ at a modulation frequency of $100 \mathrm{~Hz}$ yields 13 spectral components on each side of the carrier frequency, whose relative amplitudes are above 0.1 of the amplitude of the unmodulated carrier frequency (see, e.g., Wozencraft and Jacobs, 1967). Most energy is distributed to the frequencies around $\mathrm{f}_{o} \pm \mathrm{df}$.

Tuning curves, impulse-frequency response functions and response patterns for different frequencies were recorded for each neuron prior to stimulation with modulated sounds. With modulated signals the following properties of the neurons were determined: thresholds for modulation depth, maximum and most effective modulation rate, response patterns to SFM and SAM stimuli and dependence of the synchronized modulation response on the sound intensity and carrier frequency. 


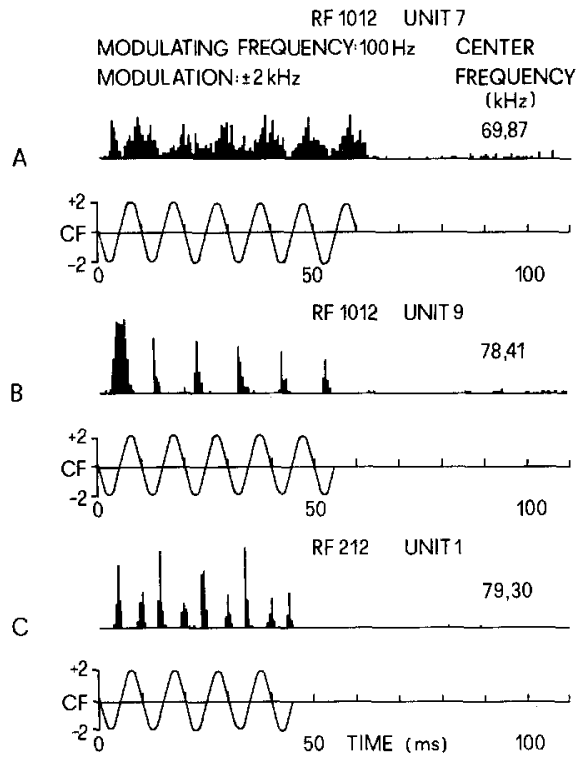

Fig. 1. A-C. Response pattern of single collicular neurons to sinusoidally frequency modulated stimuli (SFM). The upper line in each section shows the PST-histogram elicited by the SFMs as depicted in the lower line. The modulation depth was always $\pm 2 \mathrm{kHz}$, the modulation rate was $100 \mathrm{~Hz}$ and the carrier or center frequency is indicated on the right side. Each PST-histogram is composed of 40 repetitions. A SFM response of a neuron with tonic response to pure tones $\mathbf{B}$ Transient neuron responding only to downward sweep cycles of SFM, $\mathbf{C}$ Transient neuron responding to down-and up-ward sweep cycles of the SFM

\section{Results}

Upon encountering a neuron, the tuning curve and firing pattern evoked by pure tones at the BEF were determined. In agreement with Möller et al. (1978) two broad categories of firing patterns elicited by tonal signals were observed: (1) tonic or sustained responses and (2) on- or on-off- responses (transient responses). Subsequently, sinusoidally frequency modulated (SFM) stimuli, where the carrier frequency was set at the neuron's BEF, were presented. The intensities of the SFM signals were 30-50 dB above threshold of the BEF.

\section{Response Patterns}

Of the 47 neurons tested, $46(97 \%)$ responded to SFM stimuli at modulation depths equal to or smaller than $\pm 2 \mathrm{kHz}$. The BEFs of the neurons ranged from $12 \mathrm{kHz}$ to $87 \mathrm{kHz}$. There were, however, a variety of response patterns evoked by the SFM sounds. The patterns elicited by the SFM sounds were correlated, in large part, with the two categories of firing patterns evoked by tone bursts at the $\mathrm{BEF}$, i.e., tonic or transient response patterns.

All neurons displaying a tonic firing pattern to pure tones, or having a prominent sustained component following a strong on-response, had a characteristic response pattern to SFMs. As shown in Fig. 1A, the most prominent feature of these cells was a modulated tonic discharge pattern which followed the modulating frequency. Although the phase of the discharge peaks lagged with respect to the waveform, the discharge pattern resembled the sinusoidal waveform, at least for low modulation rates (smaller than $80 \mathrm{~Hz}$ ). 
Neurons exhibiting the above feature had BEFs which spanned the entire audiogram of Rhinolophus $(10-90 \mathrm{kHz})$. No particular frequency band was favored and no relation between the BEF of these cells and the frequencies in the FM or CF components of the echolocation signals were observed.

Neurons displaying an on- or on-off-pattern to pure tones responded differently to SFMs than did the sustained responders. Most transient neurons responded with only a few spikes to distinct parts of the modulation cycle (Fig. 1B and C). In these cases it is difficult to determine exactly which frequency transition triggered the neural responses. However, it seems reasonable to assume that when the carrier frequency of the SFM was set at the neuron's BEF, the discharge latencies to the SFM transitions were similar to the latency of the response to the onset of the modulated tone signal (see Fig. 1). Given this assumption, it would appear that transitions through frequencies close to the BEF are most effective for firing the neuron.

It is noteworthy that the sweep direction was an important parameter. Most neurons responded only when the signal swept downward through the carrier frequency and not to the upward transition (Fig. 1B). A smaller number of neurons fired to both the upward and downward transitions (Fig. 1C) but no neuron responded exclusively to upward transitions.

Whether a neuron responded to both sweep directions or only to downward sweeps depended largely on the modulation depth and less on the modulation rate. In some neurons responding only to downward sweeps at low modulation depth (i.e., $\pm 200 \mathrm{~Hz}$ ) discharges to both the upward and downward transitions could be evoked by increasing the modulation depth from $\pm 200 \mathrm{~Hz}$ to $\pm 1000 \mathrm{~Hz}$. Such changes, however, were not always observed since some neurons responded exclusively to downward transitions even with the largest modulation depths tested $( \pm 2000 \mathrm{~Hz}$ ).

The transient cells appear to be specific for processing echolocation sounds and echoes of the long constant frequency component at $81-88 \mathrm{kHz}$ and the final frequency modulated component at $65-81 \mathrm{kHz}$. All of the transient neurons had BEFs between 65 and $88 \mathrm{kHz}$, i.e., at frequencies occurring in the natural echolocation pulses, but no transient type neurons were found that had BEFs in the frequency range below $65 \mathrm{kHz}$.

\section{Responses as a Function of Frequency of Modulation}

In order to simulate the variety of frequency modulation patterns in the echo $\mathrm{CF}$ component produced by the wingbeats of different insect species, the modulation rate was systematically increased from $10 \mathrm{~Hz}$ to $500 \mathrm{~Hz}$. The modulation depth and carrier frequency of the signals were held constant. The carrier frequency again was at the neuron's BEF.

The discharge pattern of tonic neurons tended to follow the envelope of the modulating frequency when the modulation rate was below 80 to $100 \mathrm{~Hz}$. With increases in modulation frequency above $100 \mathrm{~Hz}$ (mimicking insects with progressively faster wingbeats), however, the discharges evoked by the SFM signals merged together (neurons + and $x$ in Fig. 3 ). Consequently, at high 


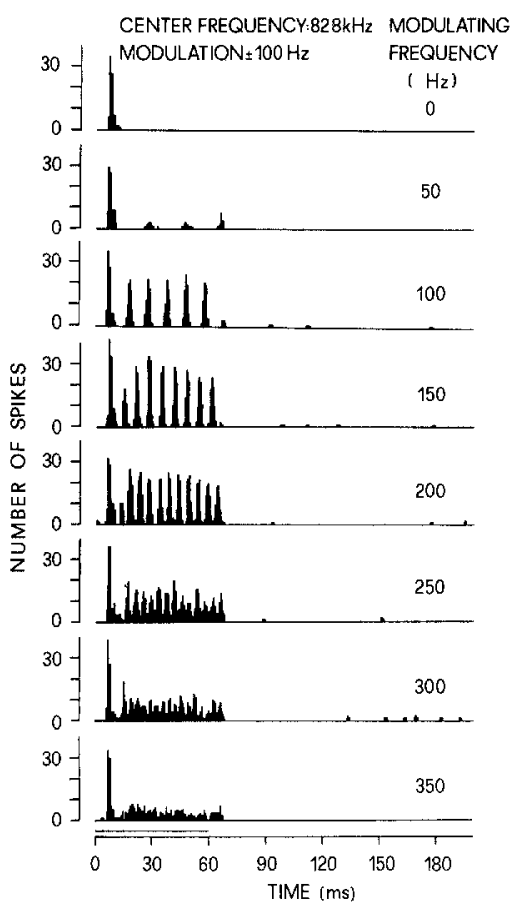

Fig. 2. SFM-responses of an inferior colliculus neuron at different modulation rates. The carrier or center frequency of the SFM was $82.8 \mathrm{kHz}$ (BEF of the neuron) and the modulation depth was $\pm 100 \mathrm{~Hz}$ (between 82.7 and $82.8 \mathrm{kHz}$ ). The modulation rate was increased in $50 \mathrm{~Hz}$ steps and is indicated on the right side of each histogram

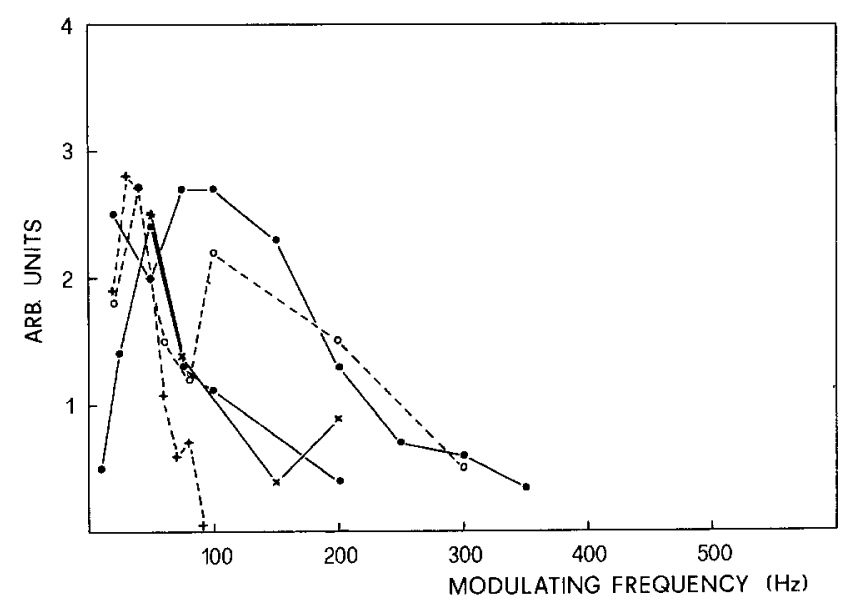

Fig. 3. Responses of 5 neurons to SFM-stimuli as a function of the modulation rate. The discharges per most effective half cycle are given in arbitrary units on the ordinate as a function of the modulation rate

modulation rates the individual peaks were no longer resolvable and the pattern became indistinguishable from the sustained pattern evoked by pure tone stimulation.

Transient responding neurons responded in a similar manner to SFM signals as the tonic neurons. In these neurons, the number of discharges per SFM-cycle 
diminished at higher modulating frequencies (Fig. 2) and in some units the responses vanished completely at high modulation rates so that only the on- or on-off responses were preserved. In 2 neurons the maximum modulation rate was 300 and 350 , respectively, while other neurons failed to follow rates higher than 100-200 Hz. The dependence of SFM-responses on the modulation rate is shown graphically in Fig. 3. In this figure the ability to follow the modulation envelope is expressed as the number of discharges per most effective half cycle. From this figure it can also be seen that modulation frequencies between $20 \mathrm{~Hz}$ and $100 \mathrm{~Hz}$ generally yielded the strongest responses to SFMs although some neurons responded with discharges well synchronized to the modulation envelope up to modulating frequencies of $200-300 \mathrm{~Hz}$.

\section{Responses to SFMs Having Different Carrier Frequencies}

The data presented above were obtained with SFM signals where the carrier frequency of the signal was set at the neuron's BEF. But it should be recalled that Horseshoe bats exhibit Doppler shift compensation where the emitted frequency (CF component) is adjusted to hold the echo CF at one particular reference frequency. As a result, it becomes of interest to inquire into neuronal responsiveness to SFMs where the carrier frequency is set at values other than the neuron's BEF. In this section the ability of neurons to encode SFMs when the carrier is shifted above and below the BEF is discussed.

Typically, the degree of response synchronization depended upon the carrier frequency of the SFM signals and the location of the carrier frequency within the neuron's tuning curve. Of particular importance is that the frequency modulation in the high frequency half of the tuning curve was optimal for evoking a modulation of the neuron's discharge pattern. Fig. 4 shows a series of SFM responses for different carrier frequencies (the modulation depth was $\pm 2 \mathrm{kHz}$ at a modulation rate of $50 \mathrm{~Hz}$ ). The graph to the left of each histogram demonstrates the location of one SFM cycle within the high and low frequency borders of the tuning curve. Several features of this figure are noteworthy. First, when the carrier frequency was $78 \mathrm{kHz}$, a frequency lower than the $\mathrm{BEF}$, the responses were poorly synchronized to the modulation waveform. This poor synchronization occurred even though the frequencies swept through the BEF. Second, as the carrier frequency of the SFM was progressively raised, first approaching and then exceeding the BEF of $79.2 \mathrm{kHz}$, the discharge pattern became highly synchronized to the modulating waveform. The envelope of the discharge pattern, however, was distorted due to the superior responsiveness to the downward sweeping portion as compared to the upward sweeping portion of the SFM signal. Third, when the carrier frequency was above $81 \mathrm{kHz}$ the neuron fired briskly only to the downward component of the sweep and failed to fire to the upward component. Note, the vigorous firings were evoked although the signal never swept through the BEF. For the $82.5 \mathrm{kHz}$ carrier frequency condition, only a small part of the high frequency side of the tuning curve was entered by the signal yet the firings to the downward component were brisk. 


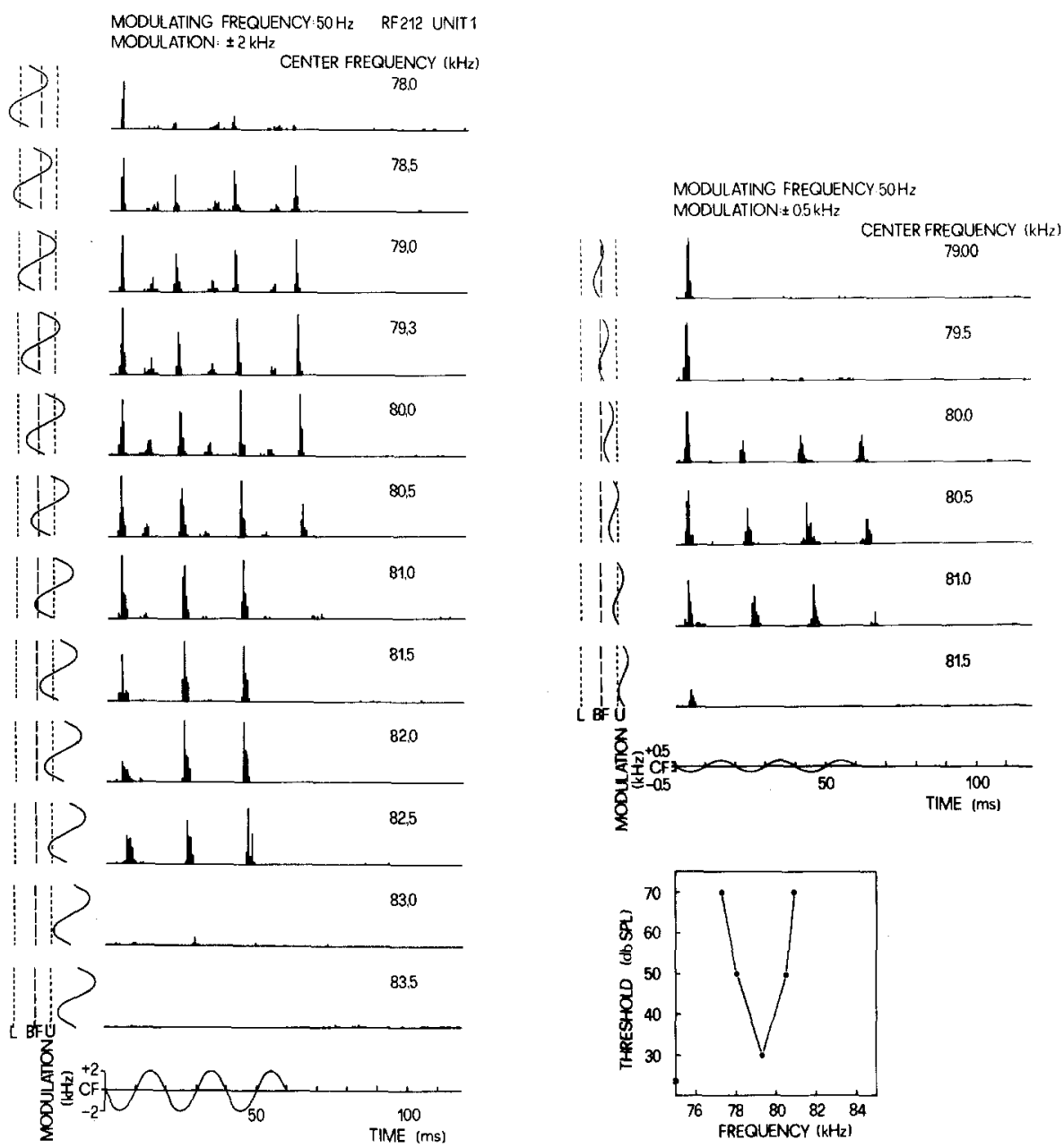

Fig. 4. Influence of the carrier or center frequency of the SFM on the response of a collicular neuron at two different modulation depths ( $\pm 2 \mathrm{kHz}$ (left) and $\pm 500 \mathrm{~Hz}$ (right)). The bottom line describes the time course of the modulation whereas the drawing to the left of each histogram depicts the position of the modulation cycle within the limits of the tuning curve at the chosen intensity of $70 \mathrm{~dB}$ SPL (L: lower threshold, BF: best excitatory frequency, U: upper threshold of the tuning curve). The inset on the lower right side shows the tuning curve of the neuron. The carrier or center frequency is indicated for each histogram at the right side. The modulation rate was always $50 \mathrm{~Hz}$

Lastly, little or no responsiveness was observed if the signal sweep lay completely outside the tuning curve.

The importance of sweeping through the high frequency side of the tuning curve is also illustrated in the right graph of Fig. 4. In this case the modulation depth was only $\pm 500 \mathrm{~Hz}$ and only responses to the downward component of the SFM cycle were evoked. But the feature of interest is that the optimal response occurred when the carrier frequency of the SFM was located in the high frequency side of the tuning curve, i.e., above the BEF. 
Fig. 5. Dependence of the minimum SFM depth eliciting a response in collicular neurons on the carrier or center frequency of the SFM-stimulus. The vertical dashed lines indicate the lower and upper limits of the tuning curve at $80 \mathrm{~dB}$ SPL

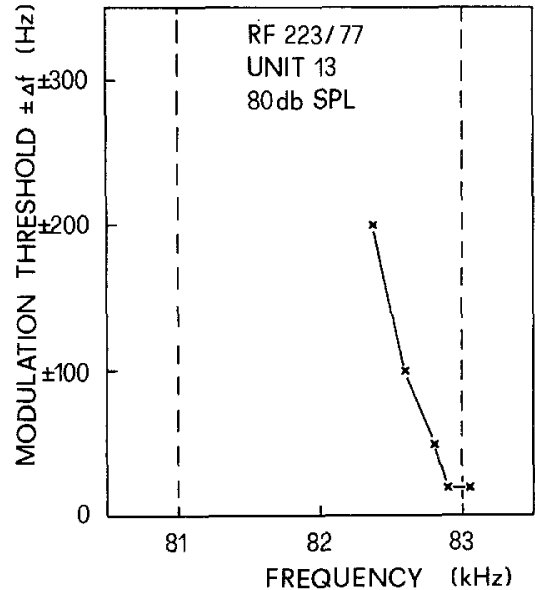

The carrier frequencies of the SFM signals also have a prominent effect on the latencies of the discharge peaks (for 2 nd and following peaks). The initial peak is most likely due to the onset of the signal and thus will not be synchronized to the SFM. As the carrier frequency is raised, the latency of the responses to the downward sweeping component increased while the latencies of the discharges evoked by the upward sweeping component decreased. The latency shifts are consistent with the response being evoked as the stimulus frequency crosses a particular frequency range in the tuning curve.

In some neurons, large modulation depth of $\pm 2 \mathrm{kHz}$ did not elicit responses when all frequencies in the cycle were lower than the BEF, even when all frequencies covered by the sweep were within the response area. The feature that downward transitions through the high frequency portion of the tuning curve (above the BEF) evoked the most vigorous synchronized discharges, and in some cases were the only SFM sounds to evoke synchronized firings, was found in all units tested.

The minimum modulation depth (modulation threshold) capable of synchronizing neural firings was also found to depend upon the carrier frequency of the SFM. As shown in Fig. 5, the modulation threshold was reduced from $\pm 200 \mathrm{~Hz}$ at $82.5 \mathrm{kHz}$, to $\pm 20 \mathrm{~Hz}$ at the high frequency border of the tuning curve which was about $83.0 \mathrm{kHz}$ (the response area at $80 \mathrm{~dB}$ is indicated by the dashed lines). Stated differently, frequency changes of only $\pm 20 \mathrm{~Hz}$ in the constant frequency component of the echo are encoded by this neuron in the form of neural discharges synchronized to the modulating waveform.

It is worth emphasizing that neurons having very low modulation thresholds (i.e., \pm 10 to $\pm 20 \mathrm{~Hz}$ ) had several other features in common. First, they all had BEFs either in the frequency range between 81 and $88 \mathrm{kHz}$ (long constant frequency component of the echolocation signal) or between 79 and 81 (frequency range of the upper part of the final FM-sweep in the echolocation signal). Second, the tuning curves were extremely narrow with $Q_{10 \mathrm{~dB}}$ values 


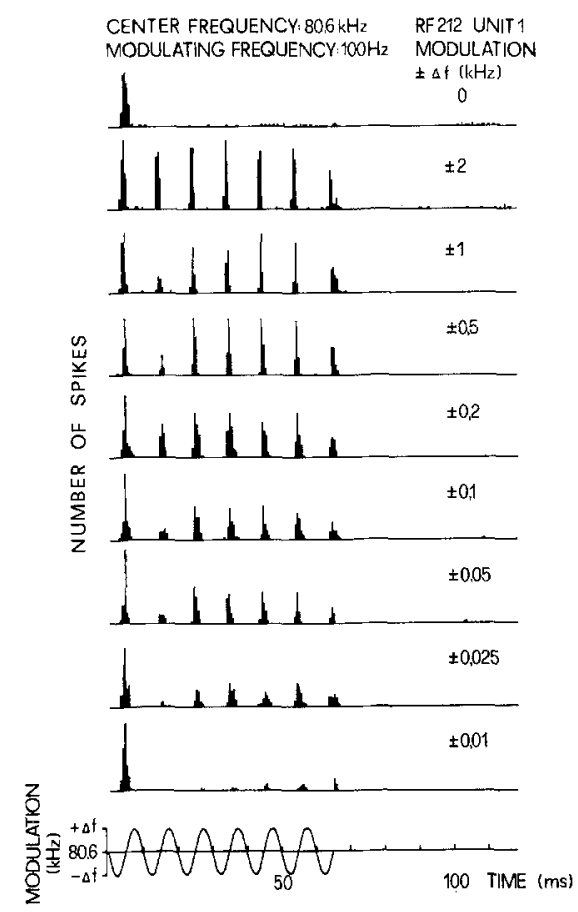

Fig. 6. Discharge patterns of a sharply tuned transient neuron to SFM-stimuli of decreasing modulation depth. The modulation rate was $100 \mathrm{~Hz}$, the carrier or center frequency was $80.6 \mathrm{kHz}$ (BEF of the neuron was $79.3 \mathrm{kHz}$ ) and the modulating waveform is drawn in the lower line. The modulation depth is indicated to the right of each histogram composed of 40 stimulus repetitions

between 42 and 240. Third, with only one exception (tonic unit), they all exhibited a transient response pattern to pure tone burst stimulation.

Fig. 6 illustrates the exquisite sensitivity of a sharply tuned transient neuron for SFM signals. The $\mathrm{Q}_{10 \mathrm{~dB}}$ of this neuron was 42 with a best frequency of $79.3 \mathrm{kHz}$. When SFM signals with a carrier frequency of $80.6 \mathrm{kHz}(1.3 \mathrm{kHz}$ above the BEF) were presented, discharge peaks were clearly synchronized to the modulation waveform at modulation depth ranging from $\pm 25 \mathrm{~Hz}$ to $\pm 2000 \mathrm{~Hz}$. This neuron responded with synchronized discharges down to a modulation depth of $\pm 10 \mathrm{~Hz}$.

The neural responses were, in general, hardly affected by the intensity of the stimulus. This parameter was tested in 5 neurons at intensities of from 10 to $50 \mathrm{~dB}$ above the BEF threshold. To be sure, the number of firings per SFM cycle increased with intensity, however, the modulation threshold was unchanged throughout the $40 \mathrm{~dB}$ intensity range and the synchronization of the neuronal discharges to supra-threshold modulation depths was conserved over the entire intensity range.

\section{Sinusoidal Amplitude Modulations (SAM)}

Under natural conditions, echoes reflected from moving targets will be both frequency and amplitude modulated with the amplitude modulations having the same modulation rate as the FM but being out of phase. Consequently, it is of 

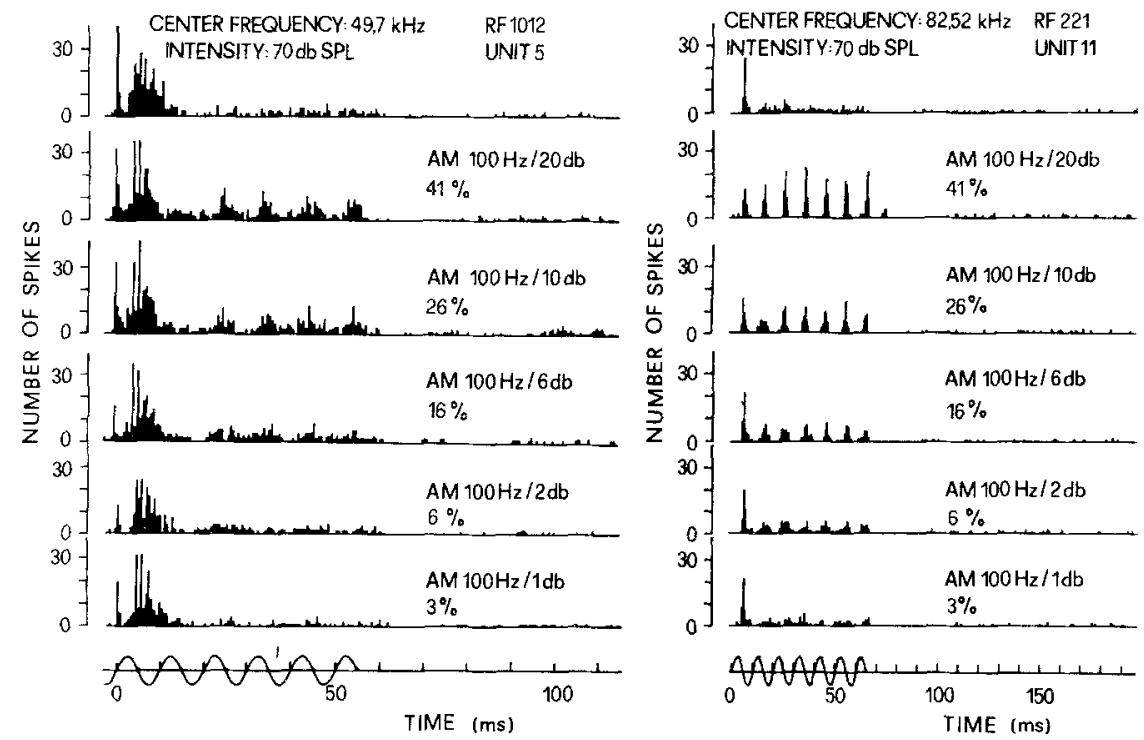

Fig. 7. Response pattern of 2 collicular neurons to sinusoidally amplitude modulated (SAM) stimuli. The frequencies of the stimuli were $49.7 \mathrm{kHz}$ and $82.5 \mathrm{kHz}$, respectively, and the carrier intensity was $70 \mathrm{~dB}$ SPL. The modulation rate was $100 \mathrm{~Hz}$. The amplitude modulation is indicated as the difference of the minimum and maximum intensities in decibels and as modulation index in percent to the right of each histogram. The upper histograms show the responses to the unmodulated sounds and the lower lines give the time course of the SAM. Each histogram is composed of 40 stimulus repetitions

interest to inquire into the response patterns evoked by AM signals. Amplitude modulations occurring under natural conditions in insect pursuit will generally not be $100 \%$ modulations but will have lower modulation indices due to the unmodulated reflections of the insect body.

\section{Response Patterns to SAM}

The SAMs were tested at the best frequencies of the neurons at intensities ranging from $15 \mathrm{~dB}$ to $40 \mathrm{~dB}$ above threshold and at modulation indices between $100 \%$ and $3 \%$. Practically every neuron tested responded to $100 \%$ SAMs with periodic discharges. This can be easily understood since a $100 \%$ modulation index corresponds to repetitive stimulation with tone bursts. The repetitive discharges, then, are a series of sequential on-responses.

Of 47 neurons tested 31 (66\%) responded to amplitude modulations when the modulation index was equal to or smaller than $41 \%$. These neurons generally showed a discharge pattern that roughly followed the modulation cycles with a latency equivalent to the on-response latency (Fig. 7). In neurons having a prominent on-response and only little sustained activity during the stimulus, this pattern was present only for lower modulation indices. With increasing modulation indices the responses sharpened and displayed clearly 
separated peaks to the transitions through the maximum amplitude (Fig. 7, right). One neuron was found that responded to both the upward and downward portions of the modulation cycle with separate responses. Unfortunately, it was not determined whether this neuron had an upper threshold which could explain the two responses to each cycle by the upward and downward transitions through the upper threshold.

\section{Thresholds for SAMs}

The minimum modulation index that evoked synchronized discharges was determined in 27 neurons. The intensity of the modulated carrier was chosen so that the relative modulations were most effective in evoking synchronized discharges and ranged between 15 and $40 \mathrm{~dB}$ above threshold. The SAMs were delivered at the same modulation rate that evoked the best responses to sinusoidally frequency modulations.

In 19 cells the threshold in terms of the modulation index was below $25 \%$ and in 8 cells below $10 \%$. The lowest threshold found was $3 \%$ modulation. Fig. 7 shows the responses of two neurons to SAMs with decreasing modulation index. The thresholds of the modulation index and the best frequencies of the neurons showed no functional relationship. The threshold for $100 \%$ modulation was reached when the maximum intensity level in the modulation cycle was at threshold intensity for the best frequency.

\section{Discussion}

Information about target structure should be obtainable from the spectral composition of the biosonar signals. There are several reasons to believe that the long CF component is particularly well suited for conveying this information. Of primary consideration is that the echoes of this component undergo three types of spectral changes: (1) an increase in the carrier frequency due to the Doppler shifts imposed by the greater flight speed of the bat relative to its target, (2) periodic frequency modulations superimposed on the carrier resulting from the additional Doppler shifts created by the movements of the insects' wings, (3) a simultaneous amplitude modulation having the same modulation or repetition rate as the frequency modulations. The equivalence of the modulation rates occurs because both the amplitude and frequency modulations are produced by the same wing beat pattern. The long duration of the CF component of the signal insures that more than one cycle of the periodic modulation will be present in the echo, e.g., an echo having a duration of 50-60 ms will have two or more modulation cycles so long as the target's wings oscillate at a frequency higher than about $20 \mathrm{~Hz}$.

The two types of neurons (i.e., tonic and transient neurons) seen in the inferior colliculus were not equally effective in their ability to encode modulations in the echo CF component. Whereas the tonic neurons responded to SFM signals with spike histograms which mirrored the modulating waveform 
of the signal, they showed this behavior only for low modulation frequencies that were typically below $80 \mathrm{~Hz}$. Consequently, tonic neurons do not seem to be capable of following the faster modulations produced by the wingbeats of most insects and hence apparently cannot encode the details of the fine structure in the echoes.

Transient neurons, on the other hand, accurately followed the sinusoidal frequency modulations. In most neurons the SFM-responses to frequency transitions were precisely synchronized for repetition rates up to $200 \mathrm{~Hz}$ and some neurons followed repetition rates up to $350 \mathrm{~Hz}$. Collicular neurons, then, apparently can resolve frequency changes which are periodically repeated every 3-5 ms. Moreover, the synchronization of responses to SFM cycles are largely independent of intensity, so that the intensity of the modulated echo has little effect on the detectability of the modulation.

The optimum modulation rate for transient neurons is between 20 and $100 \mathrm{~Hz}$ which covers most of the wing beat frequencies of the insects Rhinolophus presumably preys upon. Assuming the preferences of Rhinolophus are similar to Pteronotus parnellii, Rhinolophus should never pursue nonmoving targets and only seldomly hunt insects having wingbeats at or below $15 \mathrm{~Hz}$. At these low wing beat frequencies only one cycle of the frequency modulation can elicit a response during one sound of about $50 \mathrm{~ms}$ duration and as a consequence the oscillating character of the reflecting wings cannot be detected.

Many transient neurons had modulation depth thresholds as low as \pm 10 to $20 \mathrm{~Hz}$. It should be pointed out that the modulation depth is dependent not only upon the wing beat frequency, but also upon the size of the wings and the orientation of the insect with respect to the impinging sound. The interaction of these features is discussed below. The relevant point for the moment is that to be effective encoders of periodic frequency modulations neurons must be sensitive to a wide variety of modulation depth and the transient neurons are well qualified in this respect.

Another important feature of transient neurons is the response patterns to SAM signals. Many neurons were sensitive to small amplitude variations and responded to modulation indices of 10-20\%. Indeed, one neuron even followed amplitude changes of only $3 \%$. The high resolving power of transient neurons for both SFM and SAM signals and the fact that all transient neurons had BEFs in the frequency range between 65 and $88 \mathrm{kHz}$, the frequency range covered by the natural echolocation sound, should enable these neurons to encode the fine structure of the echoes created by wing beat patterns and hence inform the bat about attributes of its prey.

It is now well established that under laboratory conditions Rhinolophus adjusts the frequency of its emitted CF component to compensate for Doppler shifts in the echo CF component. The net effect of this Doppler shift compensation is to hold the echo CF at one particular reference frequency within a remarkably narrow range of $50 \mathrm{~Hz}$. However, this does not mean that the echoes received while hunting insects in the wild will always return at the reference frequency. Clearly this is an impossibility given the changes in fligth speed and direction of both the bat and the target. Rather, Doppler shift compensation appears to be a mechanism whereby the echo CF is maintained 
within a narrow band of frequencies, usually referred to as the filter range (upper frequencies of the final frequency sweep should be also included here). Thus, if at a given moment an echo returns at a frequency above the reference frequency the next emitted pulse is lowered and the subsequent echo frequency is reduced. The striking correlation between the behavior and neurophysiology is that the BEFs of the transient neurons correspond exactly to the frequency range of the echo $\mathrm{CF}$ component which the bat must receive while hunting insects and using Doppler shift compensation.

The differential effectiveness of downward as opposed to upward SFMs and the predominance of activity evoked when the frequency transitions enter the high side of the tuning curve may serve to confine and sharpen the locus of activity evoked by echoes having frequency modulations. For example, consider the spatial extent of activity elicited by a Doppler shifted echo having a carrier of $83 \mathrm{kHz}$ and a modulation depth of $\pm 200 \mathrm{~Hz}$. Neurons having BEFs of $83 \mathrm{kHz}$ will clearly encode the modulations faithfully. A full-blown response will be evoked in those neurons in which the frequency sweep enters the upper frequency portion of the tuning curve or just clips the tuning curve, whereas adjacent neurons with slightly lower BEFs will be silent. As the frequency sweeps upward and above the carrier, the cells with BEFs above $83 \mathrm{kHz}$ will initially not be fired. On the downward sweep, however, cells tuned above $83 \mathrm{kHz}$ will fire but only if the frequency transition encroaches upon the high frequency side of the tuning curve. Synchronized activity will be minimal in neurons where only the low frequency portion of the tuning curve is stimulated. These features, coupled with the exceptionally sharp tuning of the filter neurons, should produce a sharp boundary between cells firing synchronously to the modulated signal and the surrounding neural space.

\section{Information Extracted from Modulated Echoes}

Below, arguments are advanced which strongly suggest that transient neurons provide information that should enable the bat to discriminate among various types of insects and perhaps to uniquely identify a particular species.

\section{a) Discriminating Moving Targets from Stationary Surrounds}

The mechanism for this perceptual ability is perhaps the easiest to visualize. Moving targets will cause a Doppler shift on the carrier of the echo thereby signalling the presence of a moving target in the bat's acoustic space. The extremely sharp tuning of the neurons allows the separate populations to respond to non-Doppler shifted and Doppler shifted echoes simultaneously and thus resolve a moving target from background echoes. Flying insects can be distinguished from other moving targets by virtue of the ability of transient neurons to encode the periodic frequency and amplitude modulations introduced by wing beats on the echo carrier. 


\section{b) Prey Identification}

This is clearly a more complex perceptual task than simply discriminating a flying from an inanimate target. The frequency and amplitude modulation patterns depend upon both wing beat frequency and wing length. According to Sotavalta (1947), wing beat frequency and wing length are closely correlated for a given family where increasing wing lengths are associated with lower wing beat frequencies. Plots of wing beat frequencies against length approximate straight lines on double log coordinates and the lines are clearly separated for each insect family. Since transient and tonic neurons can encode both AM and FM the bat presumably takes advantage of all available information for prey identification.

For example consider the modulation pattern generated by two individual insects belonging to different families but having the same wing length. Since the intercepts of the beat frequency length functions differ among families, the two individuals will have different wing beat frequencies. The rates of the frequency and amplitude modulatios therefore differ and the depth of the frequency modulation produced by the faster beating wings will be much higher than the slower beating insect due to the higher velocity of the reflecting surface (i.e., the wings). On the other hand, two individuals from different families having the same wing beat frequency will have different wing lengths. Both the frequency and amplitude modulations therefore differ and the depth of the frequency modulation produced by the faster beating wings will be much higher than the produced by insects of various families due to the differences in wing frequency length ratios and the influence that these physical features have on the modulation pattern.

The acoustic images discribed above result from sounds impinging upon different insects when the orientation of the sound source with respect to the insect is held constant. However, the modulation pattern strongly depends upon the orientation of the bat with respect to its target. A possible complication for prey recognition arises because the echo modulation pattern will be radically different when the bat approaches an insect head on, for example, from the pattern occurring when it approaches the same insect on a path perpendicular to the insect's flight direction. Nevertheless, the acoustic image should still be unique to a given species for sounds hitting at a particular angle of incidence. For insect identification the bat would have to associate a given species of insect with the entire family of echoes reflected at various attitudes.

Acknowledgements. I wish to thank Dr. G. Pollak (Austin, Texas) for fruitful discussions and for the correction of the English version, as well as to Miss N. Chayegan and Miss A. Polotzek for valuable technical help.

\section{References}

Goldman, L.J., Henson, O. W., Jr.: Prey recognition and selection by the constant frequency bat, Pteronotus p. parnellii. Behav Ecol Sociobiol 2, 411-419 (1977)

Johnson, R.A., Henson, O.W., Jr., Goldman, L.R.: Detection of insect wing beats by the bat, Pteronotus parnellii. J Acoust Soc Amer 55, 53 (1974) 
Möller, J., Neuweiler, G., Zöller, H.: Response characteristics of inferior colliculus neurons of the awake CF-FM bat, Rhinolophus ferrumequinum. I. Single-tone stimulation. J Comp Physiol 125, 217-225 (1978)

Schnitzler, H.-U.: Die Ultraschall-Ortungslaute der Hufeisenfledermäuse (Chiroptera Rhinolophidae) in verschiedenen Orientierungssituationen. Z Vergl Physiol 57, 376-408 (1968)

Schuller, G.: Echoortung bei Rhinolophus ferrumequinum mit frequenzmodulierten Lauten evoked potentials im Colliculus inferior. Z Vergl Physiol 77, 306-331 (1972)

Schuller, G., Beuter, K., Schnitzler, H.-U.: Response to frequency-shifted artificial echoes in the bat, Rhinolophus ferrumequinum. J Comp Physiol 89, 275-286 (1974)

Sotavalta, O.: The flight tone (wing stroke frequency) of insects. Acta Ent Fenn 4, 5-117 (1974)

Suga, N., Jen, Ph.H.-S.: Further studies on the peripheral auditory system of 'CF-FM' bats specialized for fine frequency analysis of Doppler-shifted echoes. J Exp Biol 69, 207-232 (1977)

Wozencraft, J.M., Jacobs, I. M.: Principles of communication engineering. 2nd edition. New York: Wiley 1967

Received March 25, 1978 